\title{
Effect of Low-Level Laser Therapy and Platelet-Rich Fibrin on the Treatment of Intra-bony Defects
}

\author{
Devi Bala Raja Thalaimalai ${ }^{*}$, Dhayanand John Victor ${ }^{2}$, Ponnudurai Samuel Gnana Prakash ${ }^{3}$, Sangeetha \\ Subramaniam ${ }^{4}$, Priyanka K Cholan ${ }^{4}$ \\ 'Postgraduate, Department of Periodontics, S.R.M Dental College \& Hospital, Ramapuram, Chennai \\ 2Professor \& Head, Department of Periodontics, S.R.M Dental College \& Hospital, Ramapuram, Chennai \\ ${ }^{3}$ Professor, Department of Periodontics, S.R.M Dental College \& Hospital, Ramapuram, Chennai \\ ${ }^{4}$ Reader, Department of Periodontics, S.R.M Dental College \& Hospital, Ramapuram, Chennai
}

\section{*Correspondence to Devi Bala Raja Thalaimalai, 63, Renganathapuram, Rock street, Settiyarpatti. Thalavaipuram. Rajapalayam (tk), Virudhunagar (dt). Tamil nadu- 626122 \\ Tel: +919790352313 \\ Email: balartv@gmail.com}

Published online October 3 2020

\begin{abstract}
Introduction: Attempts to regenerate the periodontal osseous defect, which is lost as a result of periodontal disease, require the tapping of the innate healing potential of periodontium through appropriately designed therapeutic strategies. A multitude of grafted and non-grafted approaches have been used in the management of Intra-bony defects. However, they do not provide predictable periodontal regeneration. The aim of this study was to evaluate the combined effect of low-level laser therapy (LLLT) and platelet-rich fibrin (PRF), in site modulated intra-bony defects (decortication), which were accessed using a simplified papilla preservation flap (SPPF), on the clinical and radiographic outcomes of periodontal disease.

Methods: A total of 30 patients with intra-bony defects were recruited for the study and randomly distributed in two groups $(n=15)$. Test group sites were accessed with SPPF and the defects received intra-marrow Penetration (IMP) following debridement and were irradiated with a low-level laser followed by PRF grafting and suturing done. The control group defects were accessed with SPPF and grafted with PRF before being secured by sutures. The plaque and bleeding score, PPD, CAL, and the position of the gingival margin with radiographic defect depth were recorded and analyzed at baseline and six months post-intervention using the student's $t$ test and Wilcoxon signed rank test. Results: The test group showed a clinically relevant increase in mean PPD reduction, CAL gain, and radiographic bone fill $(3.6 \pm 1.35 \mathrm{~mm}, 3.26 \pm 1.16 \mathrm{~mm}$ and $2.44 \pm 1.24 \mathrm{~mm})$ compared to the control group $(2.93 \pm 1.1 \mathrm{~mm}, 2.267 \pm 1.33 \mathrm{~mm}$ and $1.26 \pm 0.99 \mathrm{~mm})$ six months post-intervention. However, intergroup comparison between the test and control groups did not show any statistically significant difference.

Conclusion: These results highlights that test protocol had greater amelioration of the effects of periodontal disease and all the investigated clinical and radiographic parameters showed considerable improvement from baseline to 6 months within test and control group, but intergroup comparison between the test and control groups did not show any statistically significant difference, indicating statistical equivalence between the test and control protocol.

Keywords: Intra-bony defect; Intra-marrow penetration; Low-level laser therapy; Platelet rich fibrin; Simplified papilla preservation flap.
\end{abstract}

\section{Introduction}

Periodontal regeneration requires the coherent tapping of the innate healing potential of periodontium through appropriately designed therapeutic strategies, which will result in the restoration of the lost periodontal architecture. The tissue engineering concept seeks to enhance regeneration through the tapping of innate progenitor cells by the utilization of biomimetic matrices that incorporate signalling molecules. ${ }^{1}$ Autologous platelet-rich fibrin (PRF) is a second-generation platelet concentrate, which is, in essence, a fibrin matrix that favours the development of angiogenesis and establishes contact inhibition at the healing periodontal defect. ${ }^{2}$ With respect to bone regeneration, PRF can be considered as a 'tissue engineering marvel' because of the ideal qualities of an osteo-promotive matrix promotes the sustained release of growth factors, that modulate and assist in osteoblastic proliferation, migration and adherence and also simultaneously up-regulating collagen related protein production. ${ }^{3}$ The above-mentioned calibre of PRF 
to provide a bioactive three-dimensional fibrin matrix enables it to function as a scaffold into which cellular elements from the grafted site might migrate to potentiate periodontal tissue regeneration.

The low-level laser has been used as an effective adjunct in periodontal regenerative surgeries in the traditional tissue engineering concept. ${ }^{4}$ It is a light-source treatment which is used to improvise the periodontal osseous defect regeneration capacities, working in the milliwatt range with wavelengths usually in the red or near-infrared spectrum (500-900 nm) without inducing thermal effects. LLLs do not cut or ablate tissues and are frequently called as a therapeutic laser or cold laser. Cold lasing has been shown to increase the proliferation of mesenchymal stem cells and enhance the upregulation of growth factors that are pertinent to periodontal regeneration. Low-level lasers further modulate signalling events to upregulate cellular proliferation. They also enhance the viability of osteoblasts through an osteogenic bio-stimulatory effect on osteoblast-like cells, thus promoting linear bone growth, thereby improving the regenerative potential of the periodontal osseous defect. ${ }^{4,5}$ The effects of low-level lasers are more significant on cellular elements. Since the cellular elements of bone, the osteocytes, are encased in mineralized lacunae in bone, this study has sought to create site modulation consisting of cortical penetrations and facilitate the intra-marrow cellular elements of osteoblasts and undifferentiated mesenchymal cells to become amenable for low-level laser modulation. In addition, SPPF has been used as a flap technique to preserve the integrity of the inter-dental gingiva during the management of the flap and to promote primary wound closure. Therefore, the test group in this study sought to assess the ability of low-level laser therapy (LLLT) of site modulated intra-bony sites (Decorticated sites) which were accessed with SPPF in order to compare the radiographic gain in bone levels, amount of clinical attachment level (CAL) gain and probing pocket depth (PPD) reduction within and between the control group and the test group from baseline to six months after the intervention.

\section{Materials and Methods}

This study was a single-blind (evaluator), randomized controlled clinical trial, conducted at the outpatient clinics of the department of periodontics and oral implantology, SRM Dental College - Ramapuram, Chennai -89. The study was conducted between June 2017 to August 2018

\section{Recruitment of Patients}

All clinical parameters were assessed by a single calibrated examiner to rule out examiner bias. Patients with intrabony defects were provisionally recruited into the study. Initially, clinical models, blood investigations and intraoral periapical radiographs were performed.

The inclusion criteria included (1) Patients with PPD $\geq 5$ $\mathrm{mm}$ and clinical attachment loss of $\geq 3 \mathrm{~mm}$; (2) Presence of 2 walled or 3 walled infra-bony defects in maxillary and mandibular posterior segments; (3) Patients with a fullmouth plaque score (FMPS $)^{6}$ and a full-mouth bleeding score $(\mathrm{FMBS})^{7}$ of $<20 \%$ after phase I therapy; (4) Evidence of $\geq 3 \mathrm{~mm}$ of intra-bony defect depth evaluated by the visualization of peri-apical radiographs.

The exclusion criteria included: (1) Patients requiring antibiotic prophylaxis before the periodontal examination; (2) Patients diagnosed with malocclusion at the site of the defect; 3) Patients with systemic disease and/or on drugs that contraindicate periodontal surgery; 4) Patients with a history of smoking and pan chewing; 5) Sites with advanced class II \& III furcation involvement.

The sample size was calculated based on the study done by Thorat et $\mathrm{al}^{8}$ in 2011 . When $\alpha$ was set at 0.05 ( $\alpha$ error $=5 \%$ ) with a power of $90 \%$, a total of 30 patients needed to be included in the study with 15 patients each in the control group and the test group. Therefore, the study included a total of 30 patients - 15 in each group. The enrolment details are presented as a flow chart in Figure 1.

All the patients were reviewed 3 to 4 weeks after phase I therapy to evaluate their oral hygiene status. Clinical examination was done using a sterile mouth mirror and a University of North Carolina-15 periodontal probe, and the following clinical parameters were assessed; (1) Full Mouth Plaque scores ${ }^{6}$ - FMPS\%, (2) Full Mouth Bleeding scores ${ }^{7}-\mathrm{FMBS} \%$, (3) PPD at the surgical site PPD $(\mathrm{mm}),{ }^{9}(4)$ relative clinical attachment level at the surgical site- RCAL (mm), ${ }^{9}$ and (5) Relative position of the gingival margin at the surgical site- PGM (mm). ${ }^{9}$

The patients who fulfilled the recruitment criteria were enrolled serially into the study. Those enrolled with even numbers were recruited into the control group and those enrolled with odd numbers were recruited as the test group. The study groups were as follows:

Group A (control group): the simplified papilla preservation flap with platelet-rich fibrin grafted into the defect site.

Group B (test group): the simplified papilla preservation flap with site modulated intra-bony defects and LLLT with platelet-rich fibrin grafted into the defect site.

\section{Site-Specific Measurements}

Site-specific measurements pertaining to the surgical site were thereafter recorded using a customized acrylic stent to ensure the accuracy of the probing site and angulation. Site-specific PPD (PPD), relative clinical attachment level (CAL), and the position of the gingival margin (PGM) from the apical level of the customized acrylic stents were thus recorded. Further, the radiographic defect depth (R/G DD) was measured from the CEJ to the base of the defect. The standardization of radiographs was done by using customized silicone bite blocks to index the dentition that was to be attached to the metal bar of the holder device and to be used to obtain reproducibility 


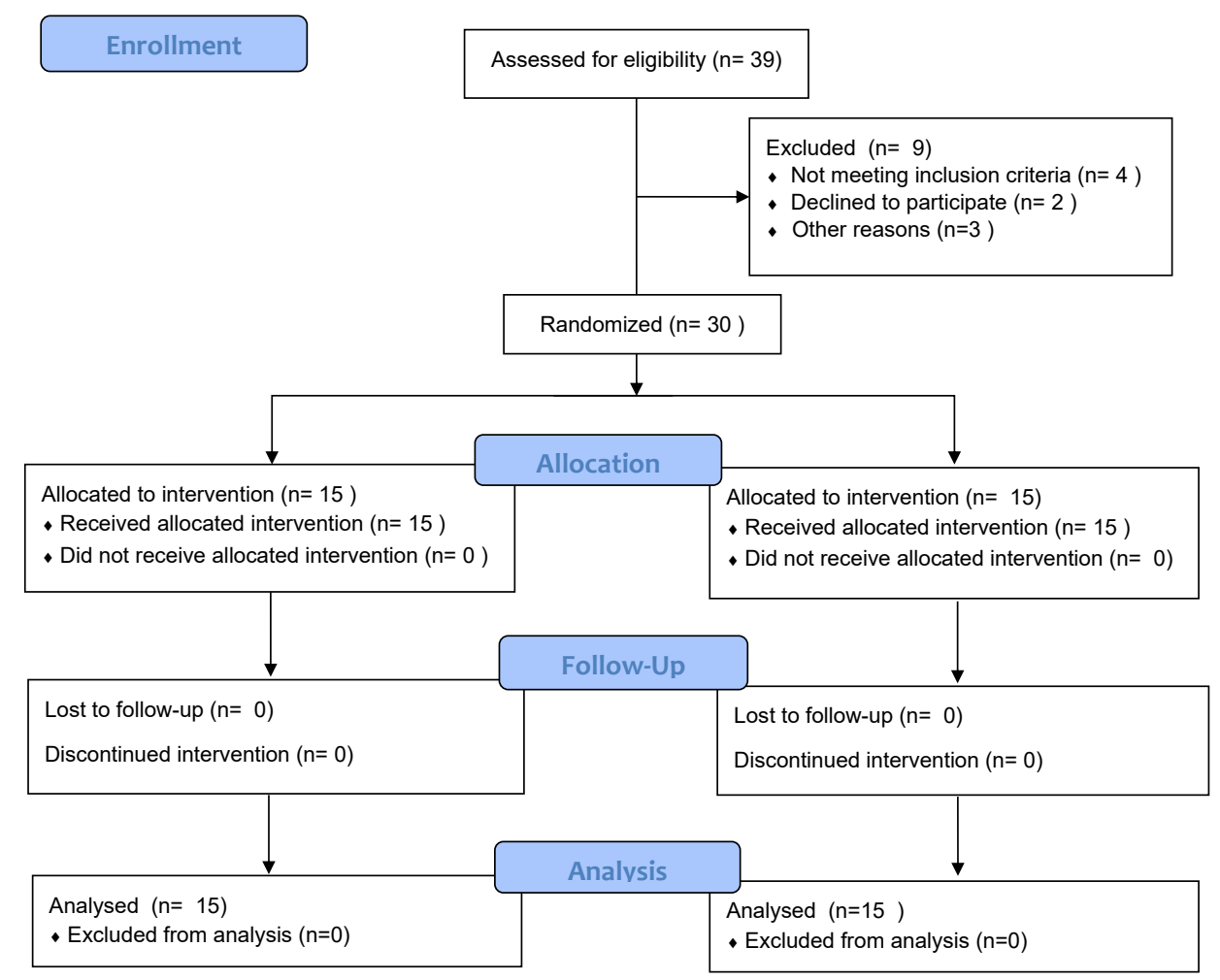

Figure 1. Enrolment Flow Chart.

using a long cone parallel technique. These digital images were analyzed using a software program, Sophix RVG 8020004 EASY DENT Software.

\section{Surgical Procedure}

After administration of 2\% lignocaine with 1:80000 epinephrine to effect local anaesthesia, both the test and control group sites were accessed with a simplified papilla preservation access flap, which was used to raise the mucoperiosteal flap associated with the defect. ${ }^{10}$ Briefly, an oblique incision was made across the papilla starting from the gingival margin at the buccal line angle of the tooth to reach the mid-interdental portion of the papilla below the contact point of the adjacent tooth. This oblique interdental incision continued intra-sulcularly along the buccal aspect of the neighbouring teeth, and full-thickness flaps were elevated. In the test sites after the SPPF access was performed, root surface debridement was meticulously performed, followed by intra-marrow penetrations (IMPs), on the defect walls using a $0.25 \mathrm{~mm}$ wide half round bur, mounted on a slow-speed handpiece. Following this, low-level laser biostimulation of the defect was effected with an $810 \mathrm{~nm}$ diode laser at $0.5 \mathrm{~W}$ power, with an uninitiated $0.6 \mathrm{~mm}$ optical fiber tip. The defects were irradiated for 20 seconds in a continuous noncontact mode and then retracted for 8 seconds. This was repeated for 3 times so that the defects were effectively lased for about 60 seconds. The defect was then packed with particulated PRF.

PRF Preparation: PRF was prepared in accordance with the protocol developed by Choukroun et al. ${ }^{2}$ PRF was prepared by collecting Intra-venous blood (from the antecubital vein) in a $10-\mathrm{ml}$ sterile glass tube without anticoagulant and immediately centrifuged in a centrifugation machine at $3000 \mathrm{rpm}$ for 10 minutes. It resulted in the separation of blood into a structured fibrin matrix in the middle of the tube, just between the red corpuscles at the bottom and acellular plasma (plateletpoor plasma) at the top. PPP was discarded. PRF was easily separated from the red corpuscles base using sterile tweezers and scissors. The junction of PRF to the Red Blood Cell layer was preserved as this region is supposed to be the richest in all the growth factors. That was further secured with PRF in the form of a membrane.

In the Control group, following the SPPF, the defect was grafted with particulated PRF, which was overlaid with a PRF membrane, without any adjunctive defect management measures. Wound closure was obtained in using vertical internal mattress sutures with Vicryl 4.0 in both groups. Periodontal dressings were thereafter placed across the wounds of both the test and control sites (Figure 2 and 3 ).

\section{Post-operative Care}

The patients were prescribed antibiotics and analgesics - amoxycillin $500 \mathrm{mg}$ and ibuprofen $400 \mathrm{mg}$ three times 

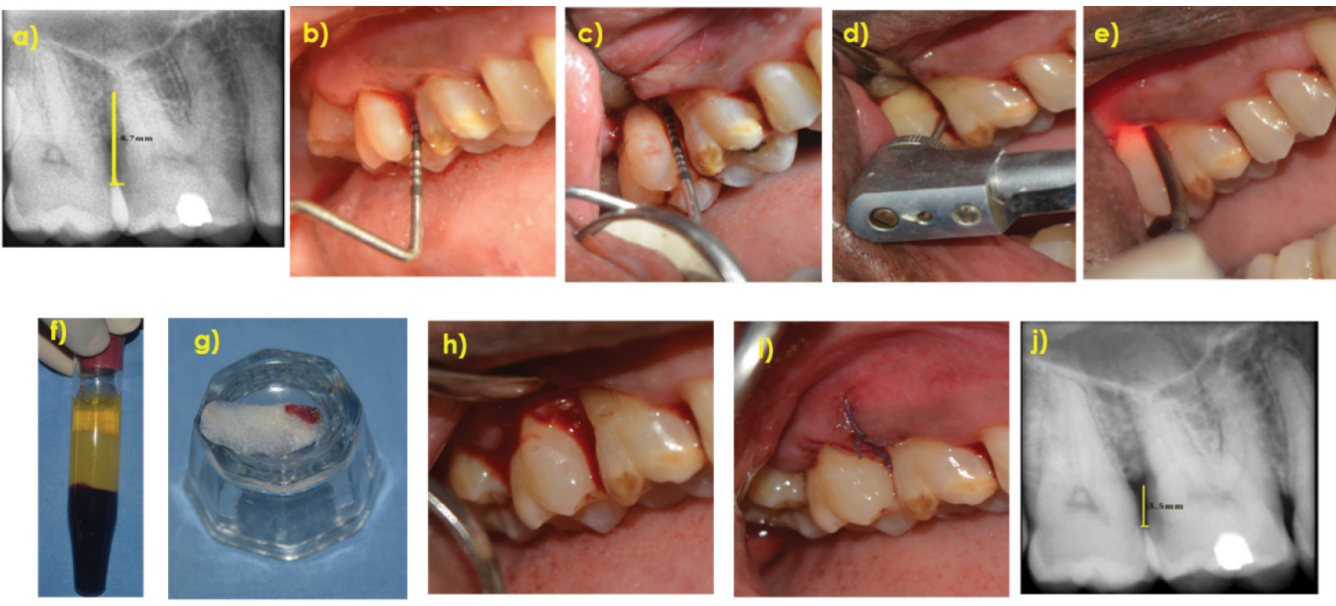

Figure 2. Surgical Protocol of Test Group (SPPF+decortication+LLLT+PRF)
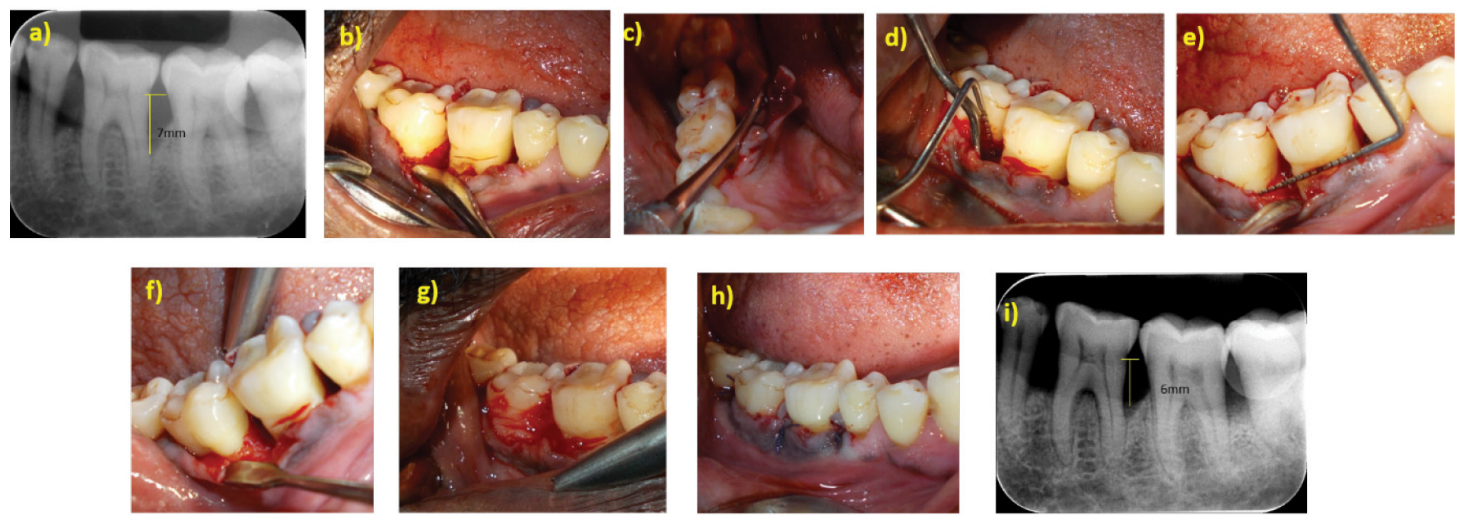

Figure 3. Surgical Protocol of Control Group (SPPF+PRF).

daily for 3 days respectively. The patients were directed to abstain from mechanical oral hygiene procedures in the surgical area for 2 weeks, and $0.2 \%$ chlorhexidine mouth rinse was used to support plaque control. All the patients were advised to report for professional plaque removal bimonthly, up to the sixth week. The patients were further advised to avoid sticky and spicy foods and hot beverages for a week. Sutures were removed two weeks after the surgery. Each patient was asked to report thereafter for a monthly maintenance regime for six months. Plaque scores and bleeding scores were evaluated at the end of the third and sixth months. Clinical parameters like PPD, CAL, PGM and radiographic defect depth were reassessed at six months. The data were collected and then subjected to statistical analysis.

\section{Results}

The parameters that are critical to the evaluation and maintenance of the intra-bony defects were assessed for all the patients. These parameters were FMPS\%, FMBS\%, the assessment of PPD, CAL, PGM and R/G DD at baseline and after 6 months. The results are expressed in Tables 1, 2, 3 and 4 .

In the statistical evaluation of the data, FMPS, FMBS, PPD, CAL and R/G DD were identified to have a normal distribution of data, and these were analysed using the Student's $t$ test. The levels of PGM did not follow a normal distribution, and this data was analysed for a significant difference between the test and control sites using the Wilcoxon signed rank test. The test group showed a clinically relevant increase in mean PPD reduction, CAL gain and radiographic bone fill $(3.6 \pm 1.35 \mathrm{~mm}, 3.26 \pm$ $1.16 \mathrm{~mm}$ and $2.44 \pm 1.24 \mathrm{~mm}$ ) compared to the control group $(2.93 \pm 1.1 \mathrm{~mm}, 2.267 \pm 1.33 \mathrm{~mm}$ and $1.26 \pm 0.99$ $\mathrm{mm})$ at six months post-intervention. The intragroup comparison of critical surrogates of periodontal regeneration viz. PPD, CAL, and $\mathrm{RDD}$, all showed statistically highly significant improvement $(P \leq 0.0001)$ at six months post-intervention, in comparison with the baseline levels in both the test and control groups. The intra-group comparison of PGM in both test and control groups showed a statistically significant apical shift in the 
Table 1. Intergroup Comparison of Clinical and Radiographic Parameters at Baseline and After 6 Months

\begin{tabular}{|c|c|c|c|c|c|c|}
\hline \multirow[b]{2}{*}{ Parameters } & \multicolumn{3}{|c|}{ Baseline } & \multicolumn{3}{|c|}{ Six Months } \\
\hline & $\begin{array}{c}\text { Test } \\
(\text { Mean } \pm \text { SD })\end{array}$ & $\begin{array}{c}\text { Control } \\
(\text { Mean } \pm \text { SD })\end{array}$ & $P$ Value & $\begin{array}{c}\text { Test } \\
(\text { Mean } \pm \text { SD) }\end{array}$ & $\begin{array}{c}\text { Control } \\
(\text { Mean } \pm S D)\end{array}$ & $P$ Value \\
\hline FMPS\% & $17.60 \pm 1.724$ & $17.80 \pm 1.971$ & 0.770 & $20 \pm 3.423$ & $21.13 \pm 3.021$ & 0.345 \\
\hline FMBS\% & $15.67 \pm 2.637$ & $16 \pm 2.449$ & 0.722 & $15.73 \pm 3.284$ & $17.87 \pm 3.99$ & 0.091 \\
\hline $\mathrm{PPD}(\mathrm{mm})$ & $7.53 \pm 1.125$ & $7.07 \pm 1.033$ & 0.247 & $3.93 \pm 0.799$ & $4.13 \pm 0.640$ & 0.455 \\
\hline $\mathrm{CAL}(\mathrm{mm})$ & $9.07 \pm 1.438$ & $8.73 \pm 1.387$ & 0.523 & $5.8 \pm 1.568$ & $6.47 \pm 1.362$ & 0.216 \\
\hline PGM (mm) & $1.53 \pm 0.915$ & $1.67 \pm 0.900$ & 0.691 & $1.87 \pm 1.060$ & $2.33 \pm 1.175$ & 0.263 \\
\hline $\mathrm{R} / \mathrm{G} \mathrm{DD}(\mathrm{mm})$ & $5.93 \pm 1.752$ & $4.42 \pm 1.675$ & 0.023 & $3.487 \pm 1.008$ & $3.160 \pm 1.135$ & 0.412 \\
\hline
\end{tabular}

FMPS, full mouth plaque score; FMBS, full mouth plaque score; PPD, probing pocket depth; CAL, clinical attachment loss; PGM, position of gingival margin; R/G DD, radiographic defect depth.

Table 2. Intragroup Comparison of the Plaque Score and the Bleeding Score in Baseline, 3 Months and 6 Months in the Test and Control Groups

\begin{tabular}{|c|c|c|c|c|c|c|c|c|c|c|c|c|}
\hline & \multicolumn{6}{|c|}{ Test Group } & \multicolumn{6}{|c|}{ Control Group } \\
\hline & Baseline & 3 Months & $\begin{array}{c}P \\
\text { value }\end{array}$ & 3 Months & 6 Months & $\begin{array}{c}P \\
\text { value }\end{array}$ & Baseline & 3 Months & $\begin{array}{c}P \\
\text { value }\end{array}$ & 3 Months & 6 Months & $\begin{array}{c}P \\
\text { Value }\end{array}$ \\
\hline FMPS\% & $17.60 \pm 1.724$ & $20 \pm 3.162$ & 0.006 & $20 \pm 3.162$ & $20 \pm 3.432$ & 1.000 & $17.80 \pm 1.971$ & $19 \pm 3.140$ & 0.073 & $19 \pm 3.140$ & $21.13 \pm 3.021$ & $0.003^{*}$ \\
\hline FMBS\% & $15.67 \pm 2.637$ & $16.40 \pm 2.947$ & 0.308 & $16.40 \pm 2.947$ & $15.73 \pm 3.283$ & 0.525 & $16 \pm 2.449$ & $16 \pm 3.485$ & 1.000 & $16 \pm 3.485$ & $17.87 \pm 3.399$ & 0.114 \\
\hline
\end{tabular}

Table 3. Intragroup Comparison of Clinical and Radiographic Parameters in the Test and Control Groups From Baseline to 6 Months

\begin{tabular}{|c|c|c|c|c|c|c|}
\hline \multirow[b]{2}{*}{ Parameters } & \multicolumn{3}{|c|}{ Baseline } & \multicolumn{3}{|c|}{ Six Months } \\
\hline & $\begin{array}{c}\text { Test } \\
(\text { Mean } \pm \text { SD })\end{array}$ & $\begin{array}{c}\text { Control } \\
(\text { Mean } \pm S D)\end{array}$ & $P$ Value & $\begin{array}{c}\text { Test } \\
(\text { Mean } \pm \text { SD })\end{array}$ & $\begin{array}{c}\text { Control } \\
(\text { Mean } \pm S D)\end{array}$ & $P$ Value \\
\hline FMPS\% & $17.6 \pm 1.724$ & $20 \pm 3.423$ & $\leq 0.008^{*}$ & $17.80 \pm 1.971$ & $21.13 \pm 3.021$ & $\leq 0.0001^{* *}$ \\
\hline FMBS\% & $15.67 \pm 2.637$ & $15.73 \pm 3.283$ & 0.932 & $16 \pm 2.449$ & $17.81 \pm 3.399$ & $0.045^{*}$ \\
\hline PPD (mm) & $7.53 \pm 1.125$ & $3.93 \pm 0.799$ & $\leq 0.0001^{* *}$ & $7.07 \pm 1.033$ & $4.13 \pm 0.640$ & $\leq 0.0001^{* *}$ \\
\hline $\mathrm{CAL}(\mathrm{mm})$ & $9.07 \pm 1.438$ & $5.80 \pm 1.568$ & $\leq 0.0001^{* *}$ & $8.73 \pm 1.387$ & $6.47 \pm 1.302$ & $\leq 0.0001^{* *}$ \\
\hline PGM (mm) & $5.93 \pm 1.752$ & $3.487 \pm 1.008$ & $\leq 0.0001^{* *}$ & $4.42 \pm 1.675$ & $3.160 \pm 1.135$ & $\leq 0.0001^{* *}$ \\
\hline $\mathrm{R} / \mathrm{G} \mathrm{DD}(\mathrm{mm})$ & $5.93 \pm 1.752$ & $4.42 \pm 1.675$ & 0.023 & $3.487 \pm 1.008$ & $3.160 \pm 1.135$ & 0.412 \\
\hline
\end{tabular}

* Statistically significant; ** Statistically highly significant.

FMPS, full mouth plaque score; FMBS, full mouth plaque score; PPD, probing pocket depth; CAL, clinical attachment loss; PGM, position of gingival margin; R/G DD, radiographic defect depth.

PGM from baseline to six months after the intervention. The inter-group comparison showed greater reduction and gain of clinical and radiographic parameters in the test group in comparison with the control group, which was not statistically significant at six months postintervention.

\section{Discussion}

The present study was designed as a randomized controlled clinical trial, which evaluated the clinical effectiveness of LLLT and PRF in site modulated intra-bony defects accessed with SPPF. The effects of this protocol on the regeneration of the periodontium were assessed through the evaluation of surrogates of periodontal regeneration, namely PPD, relative CAL and the position of the gingival margin and the radiographic defect depth at baseline and six months after surgical management.

The reduction in residual PPD is the most important clinical surrogate evaluated by periodontal surgeries. The PPD was compared at baseline and after six months for both groups. In the test and control groups, there was a highly significant reduction in PPD with the mean difference of $3.6 \pm 1.352 \mathrm{~mm}$ for the test group and $2.93 \pm$ $1.1 \mathrm{~mm}$ for the control group from baseline to six months post-intervention. When comparing PPD between groups, there was a higher mean improvement in the test group, which was not statistically significant at six months after the intervention.

This PPD reduction in the test group and the control group is significantly higher when compared with the study done by Joseph et $\mathrm{al}^{11}$ who analysed the effect of PRF in intra-bony defects and the results showed a mean 
Table 4. Intragroup Comparison of the Position of the Gingival Margin in the Test and Control Groups From Baseline to 6 Months

\begin{tabular}{lccl}
\hline PGM & Mean Rank & Z Value & P Value \\
\hline Test group & 4 & -2.236 & $0.025^{*}$ \\
Control group & 4 & -2.530 & $0.011^{*}$ \\
\hline
\end{tabular}

PGM, position of gingival margin.

*Statistically significant.

improvement of $2.27 \pm 0.29 \mathrm{~mm}$ in case of PPD after 12 months of surgical management. The reduction of PPD in the test and control sites is comparable and slightly lesser in the current study compared with the study done by Pradeep et $\mathrm{al},{ }^{12}$ who reported a mean PD reduction of $3.77 \pm 1.19 \mathrm{~mm}$ with PRF alone, showing that PRF is beneficial as a sole grafting material. The current study reveals a greater mean $\mathrm{PPD}$ reduction within the test group (SPPF+IMP+LLLT+PRF) than the control group (SPPF+PRF), which highlights the probable additional benefit of LLLT and IMP in PPD reduction. Kreisler et $\mathrm{al}^{13}$ also stated that LLLT had a stimulatory effect on PDL fibroblast proliferation, caused by the stimulation of the production of the basic fibroblast growth factor. Clinically, these properties of LLLT help to stimulate the soft tissue seal around the tooth.

However, gain in CAL is a more reliable surrogate marker for periodontal regeneration to analyse the success of periodontal surgeries. In the test group and the control group, there was a highly significant CAL gain on comparing baseline CAL levels to six months CAL level. The mean gain in CAL in the test group was 3.26 \pm 1.163 $\mathrm{mm}$, whereas in the control group the mean gain in CAL was $2.267 \pm 1.335$. This indicates once again the significant clinical reduction by the test protocol of LLLT + IMP site modulation. The lack of statistical significance is due to the large standard deviation in the results, which is due to the outliers in the population. The results in this study correlate with the results in the study done by Pradeep et al, ${ }^{12}$ which showed a CAL gain of $3.31 \pm 1.76 \mathrm{~mm}$ after 9 months.

The results in the current study also correlate with the study by Joseph et al, ${ }^{11}$ which showed a CAL gain of 3.33 $\pm 0.35 \mathrm{~mm}$ of CAL after 12 months, when PRF was used as the sole grafting material in the management of intrabony defect management, which is almost comparable to our study results. The CAL gain was significantly higher in the current study when compared with the mean CAL gain reported in a systematic review by Shah et al ${ }^{14}$ who reported only $0.95 \mathrm{~mm}$ of CAL gain when PRF is utilized as a sole grafting material. The CAL gain in the test group was comparatively more than the control group, implying a positive contribution by the cell stimulative and proliferative capacity of LLLT with the adjunctive utilization of IMP along with the three-dimensional scaffold PRF within a simplified papilla preservation access flap.
Periodontal surgeries for pocket therapy often result in gingival recession which is an unfavourable outcome affecting the patient's aesthetics and function. In this study, this remained true, with a mean gingival recession in the test sites of $0.3 \mathrm{~mm}$, whereas the control site had a mean gingival recession of $0.6 \mathrm{~mm}$. This negative shift in the position of the gingival margin in the test and control groups showed a significant increase from baseline to six months post-intervention, which was statistically significant. However, the mean negative shift in the position of the gingival margin of $0.3 \mathrm{~mm}$ in the test group may not be clinically significant.

Studies by Pradeep et al, ${ }^{12}$ Ajwani et $a l,{ }^{15}$ and Sharma et $\mathrm{al}^{9}$ showed no significant change in the gingival margin level after 9 months of post-surgical management of intra-bony defects with PRF. The current study showed a slightly greater apical shift in the gingival margin level compared to the other studies, which may be due to the influence of the multiple other risk factors for gingival recession, such as biotype, the width of attached gingiva, minimal facial bone thickness, and the utilization of a highly technique sensitive surgical procedure like SPPF that was utilized in the study.

The improvement in the above-discussed parameters of periodontal disease was further reflected in the linear bone growth at the crest of the inter-proximal bone. Within the test and control groups, there was a highly significant difference with a mean difference of $2.44 \pm 1.24 \mathrm{~mm}$ in the test group and of $1.26 \pm 0.9912 \mathrm{~mm}$ in the control group from baseline to six months after the intervention. However, intergroup comparison of radiographic defect depth showed no significant difference six months after the intervention. This study revealed a significantly higher increase in the radiographic bone fill, compared with the study by Joseph et $\mathrm{al}^{11}$ who used PRF as a sole grafting material in intra-bony defect management. Whereas the study by Joseph et $\mathrm{al}^{11}$ showed that the mean radiographic bone fill was around $1.29 \pm 0.32 \mathrm{~mm}$, a study by Thorat et $\mathrm{al}^{8}$ presented a mean bone fill of $2.12 \pm 0.69 \mathrm{~mm}$ after 9 months of surgical management. The current study revealed the radiographic bone fill of $2.44 \pm 1.24 \mathrm{~mm}$ in the test group which highlights that the beneficial effect of adjunctive use of LLLT + IMP on the management of intra-bony defect in amalgamation with PRF.

Cold Lasing causes the activation of intracellular or extracellular photo-absorbable molecules, thereby initiating intra-cellular signalling through cell signalling pathways such as p38, MAPK/ERK pathways which stimulate osteoblastic differentiation, followed by BMP/ SMAD signalling pathways, thereby producing signals that promote osteoblastic proliferation. ${ }^{16,17}$ LLLT further promotes the entry of $\beta$-Catenin into the nucleus and thus it upregulates the Wnt pathway, which further stimulates osteoblastic differentiation fostering bone formation and inhibits osteoclastic differentiation, thereby causing the 
cessation of bone resorption. Thus, LLLT of the bone has been postulated to result in increased osteogenesis, bone repair and wound healing by promoting the osteoblastic differentiation and proliferation. ${ }^{18}$

Shah et $\mathrm{al}^{14}$ in their systematic review of PRF in intrabony defect management showed a standardized mean difference of $2.33 \mathrm{~mm}$ of bone fill (1.43-3.23) after the treatment of IBDs with PRF when compared to OFD alone. In accordance with this systematic review, the current study showed a much greater increase in the mean bone fill of $2.44 \pm 1.24 \mathrm{~mm}$ in the test group and $1.26 \pm 0.99 \mathrm{~mm}$ in the control group. In our study, the better clinical outcome within the test group is probably due to the augmentative effects of the IMP of the residual bony walls of two and three wall intra-bony defects using the half-round bur of $0.25 \mathrm{~mm}$ in diameter along with LLLT and PRF.

From the results of the study, it is observed that the $\mathrm{SPPF}+\mathrm{PRF}$ group and the SPPF+IMP+LLLT+PRF group both had statistically highly significant clinical and radiological improvements from baseline to six months post-intervention within the groups; however, in the inter-group comparison, the differences between the groups were not statistically significant.

In future, multi-center studies with larger sample size and elongated follow-up periods, with further addition of SPPF alone as a separate group, will enhance the strength of this randomized controlled clinical trial.

\section{Conclusion}

This study concludes that within the limits of this study, the utilization of LLLT along with PRF in the site modulated intra-bony defects caused an improvement in the clinical and radiographic outcomes, but there was no statistical difference when comparing the test protocol to the SPPF+PRF alone in the management of intra-bony defects. Further studies with larger sample size and a long follow-up period may substantiate the effect of cold lasing of site-modulated intra-bony defects on the management of periodontal intra-osseous defects.

\section{Ethical Considerations}

The study proposal was placed before the institutional scientific and ethical review board and prior approval was procured before the commencement of the study (IRB Approval no-SRMDC/IRB/2016/MDS/No.508). All the patients participating in the study were fully explained about the nature of the periodontal disease-related defects, the surgical procedure and their possible complications, following which written informed consent was obtained.

\section{Conflict of Interests}

The authors declare no conflict of interest.

\section{References}

1. Dabra S, Chhina K, Soni N, Bhatnagar R. Tissue engineering in periodontal regeneration: A brief review. Dent Res J (Isfahan). 2012;9(6):671-680.

2. Dohan DM, Choukroun J, Diss A, Dohan SL, Dohan AJJ, Mouhyi J, et al. Platelet-rich fibrin (PRF): a secondgeneration platelet concentrate. Part I: technological concepts and evolution. Oral Surg Oral Med Oral Pathol Oral Radiol Endod. 2006;101(3):e37-44. doi: 10.1016/j. tripleo.2005.07.008.

3. Schär MO, Diaz-Romero J, Kohl S, Zumstein MA, Nesic D. Platelet-rich concentrates differentially release growth factors and induce cell migration in vitro. Clin Orthop Relat Res. 2015;473(5):1635-43. doi: 10.1007/s11999-0154192-2.

4. Hamajima S, Hiratsuka K, Kiyama-Kishikawa M, Tagawa T, Kawahara M, Ohta M, et al. Effect of low-level laser irradiation on osteoglycin gene expression in osteoblasts. Lasers Med Sci. 2003;18(2):78-82. doi: 10.1007/s10103003-0255-9.

5. Cortellini P, Tonetti MS. Minimally invasive surgical technique and enamel matrix derivative in intra-bony defects. I: Clinical outcomes and morbidity. J Clin Periodontol. 2007;34(12):1082-8. doi: 10.1111/j.1600051X.2007.01144.x.

6. O'Leary TJ, Drake RB, Naylor JE. The plaque control record. J Periodontol. 1972;43(1):38-40. doi: 10.1902/ jop.1972.43.1.38.

7. Ainamo J, Bay I. Problems and proposals for recording gingivitis and plaque. Int Dent J. 1975;25:229-35.

8. Thorat M, Pradeep AR, Pallavi B. Clinical effect of autologous platelet-rich fibrin in the treatment of intra-bony defects: a controlled clinical trial. J Clin Periodontol. 2011;38(10):925-32. doi: 10.1111/j.1600051X.2011.01760.x.

9. Sharma A, Pradeep AR. Treatment of 3-wall intrabony defects in patients with chronic periodontitis with autologous platelet-rich fibrin: A randomized controlled clinical trial. J Periodontol. 2011;82(12):1705-12. doi: 10.1902/jop.2011.110075.

10. Tonetti MS, Cortellini P, Suvan JE, Adriaens P, Baldi C, Dubravec D, et al. Generalizability of the added benefits of guided tissue regeneration in the treatment of deep intrabony defects. Evaluation in a multi-center randomized controlled clinical trial. J Periodontol. 1998;69(11):1183-92. doi: 10.1902/jop.1998.69.11.1183.

11. Joseph VR, Raghunath A, Sharma N. Clinical effectiveness of autologous platelet rich fibrin in the management of infrabony periodontal defects. Singapore Dent $J$. 2012;33(1):5-12. doi: 10.1016/j.sdj.2012.10.003.

12. Pradeep AR, Rao NS, Agarwal E, Bajaj P, Kumari M, Naik SB. Comparative evaluation of autologous platelet-rich fibrin and platelet-rich plasma in the treatment of 3-wall intrabony defects in chronic periodontitis: a randomized controlled clinical trial. J Periodontol. 2012;83(12):1499507. doi: 10.1902/jop.2012.110705.

13. Kreisler M, Christoffers AB, Willershausen B, d'Hoedt B. Effect of low-level GaAlAs laser irradiation on the proliferation rate of human periodontal ligament fibroblasts: an in vitro study. J Clin Periodontol. 2003;30(4):353-8. doi: 10.1034/j.1600-051x.2003.00001.x.

14. Shah M, Deshpande N, Bharwani A, Nadig P, Doshi V, Dave D. Effectiveness of autologous platelet-rich fibrin in the 
treatment of intra-bony defects: A systematic review and meta-analysis. J Indian Soc Periodontol. 2014;18(6):698704. doi: 10.4103/0972-124X.147400.

15. Ajwani H, Shetty S, Gopalakrishnan D, Kathariya R, Kulloli A, Dolas RS, et al. Comparative evaluation of plateletrich fibrin biomaterial and open flap debridement in the treatment of two and three wall intrabony defects. J Int Oral Health. 2015;7(4):32-7.

16. Kiyosaki T, Mitsui N, Suzuki N, Shimizu N. Low-level laser therapy stimulates mineralization via increased Runx2 expression and ERK phosphorylation in osteoblasts. Photomed Laser Surg. 2010;28(Suppl 1):S167-72. doi: 10.1089/pho.2009.2693.

17. Aleksic V, Aoki A, Iwasaki K, Takasaki AA, Wang CY, Abiko Y, et al. Low-level Er: YAG laser irradiation enhances osteoblast proliferation through activation of MAPK/ERK. Lasers Med Sci. 2010;25(4):559-69. doi: 10.1007/s10103010-0761-5.

18. Zhang RF, Wang Q, Zhang AA, Xu JG, Zhai LD, Yang XM, et al. Low-level laser irradiation promotes the differentiation of bone marrow stromal cells into osteoblasts through the APN/Wnt/B-catenin pathway. Eur Rev Med Pharmacol Sci. 2018;22(9):2860-8. doi: 10.26355/eurrev_201805_14988. 